\title{
Pleural Plaque Profiles on the Chest Radiographs and CT Scans of Asbestos-exposed Japanese Construction Workers
}

\author{
Momen ELSHAZLEY ${ }^{1,9 *}$, Eiji SHIBATA ${ }^{2}$, Naomi HISANAGA ${ }^{3}$, Gaku ICHIHARA ${ }^{4}$, \\ Ashraf A. EWIS ${ }^{5}$, Michihiro KAMIJIMA' ${ }^{6}$, Sahoko ICHIHARA ${ }^{7}$, Kiyoshi SAKAI ${ }^{8}$, \\ Mitsuo SATO ${ }^{1}$, Masashi KONDO ${ }^{1}$ and Yoshinori HASEGAWA ${ }^{1}$ \\ ${ }^{1}$ Department of Respiratory Medicine, Nagoya University Graduate School of Medicine, Nagoya 466-8550, \\ Japan \\ ${ }^{2}$ Department of Health and Psychosocial Medicine, Aichi Medical University School of Medicine, Nagakute \\ 480-1195, Japan \\ ${ }^{3}$ Center for Campus Health and Environment, Aichi University of Education, Kariya 448-8542, Japan \\ ${ }^{4}$ Department of Occupational and Environmental Health, Nagoya University Graduate School of Medicine, \\ Nagoya 466-8550, Japan \\ ${ }^{5}$ Department of Occupational Health and Industrial Medicine, Faculty of Medicine, El-Minia University, \\ El-Minia 61111, Egypt \\ ${ }^{6}$ Department of Occupational and Environmental Health, Nagoya City University Graduate School of \\ Medical Science, Nagoya 467-8601, Japan \\ ${ }^{7}$ Graduate School of Regional Innovation Studies, Mie University, Tsu 514-8507, Japan \\ ${ }^{8}$ Environmental Health Department, Nagoya City Public Health Research Institute, Nagoya 467-8615, Japan \\ ${ }^{9}$ Department of Industrial Medicine and Occupational Diseases, Faculty of Medicine, Sohag University, \\ Sohag 82524, Egypt
}

Received December 20, 2010 and accepted July 1, 2011

Published online in J-STAGE August 9, 2011

\begin{abstract}
Pleural plaques are asymptomatic focal thickenings of the pleura and considered the hallmark of asbestos exposure. However, it is often difficult to detect pleural plaques on chest $\mathrm{x}$-rays (CXR). In a retrospective study, using chest CT scans of 140 Japanese asbestos-exposed construction workers who have probable or definite findings of pleural plaque on CXR; firstly, we proposed plaque morphology-based classification for CXR findings, and then we examined if those classified findings could be confirmed as pleural plaques on CT scans. Our morphology-based classification of pleural plaque findings included nine types. The percentages of confirmed pleural plaques on CT scans by type (number of confirmed pleural plaque on CT/number of observed on CXR) were $93 \%$ (40/43) for straight, $89 \%(56 / 63)$ for diamond, $\mathbf{8 8 \%}(7 / 8)$ for double, $83 \%(19 / 23)$ for tapered medially, $80 \%(20 / 25)$ for parallel, $77 \%(23 / 30)$ for crescent, $79 \%(11 / 14)$ for tenting, $72 \%(18 / 25)$ for tapered-laterally (long type), and $0 \%(0 / 9)$ for tapered-laterally (short type). When added to the ILO classification, morphology-based classification of CXR pleural plaque findings makes its detection easier and hence chest radiograph continues to be a suitable tool for screening asbestos-related pleural plaques based on its simplicity, low radiation exposure, wide availability and cost-effectiveness.
\end{abstract}

Key words: Pleural plaques, Construction workers, Chest radiograph, CT scan

*To whom correspondence should be addressed.

E-mail: momenhafez79@yahoo.com 


\section{Introduction}

In the construction industry, asbestos is found in installed products such as sprayed-on fireproofing, fire-resistant drywall, roofing material, floor tiles, and cement pipes. In Japan, exposure to asbestos likely occurred during construction, renovation or demolition of buildings containing asbestos.

Comparing with asbestosis, a lower inhaled fiber burden results in pleural plaques ${ }^{1)}$. There is a long latency period from 20 to $30 \mathrm{yr}$ between initial exposure to asbestos and the development of pleural plaques. Pleural plaques are the commonest radiographic manifestations of asbestos exposure and the presence of bilateral scattered calcified pleural plaques can be regarded as virtually pathognomonic of asbestos exposure $^{2)}$

Histopathologically, pleural plaques are circumscribed and discrete areas of hyaline or calcified fibrosis localized in the parietal pleura of the lateral chest wall, the diaphragm, and/or the mediastinum ${ }^{3)}$.

Pleural plaques tend to be found adjacent to relatively rigid structures such as the ribs, vertebral column and the tendinous portion of the diaphragm. According to radiographic studies, the characteristic sites of pleural plaques include the posterolateral chest wall between the seventh and tenth ribs, the lateral wall between the sixth and ninth ribs, the dome of the diaphragm and the mediastinal pleura particularly over the pericardium ${ }^{4}$. The sensitivity of the plain chest x-ray (CXR) in detecting pleural plaques depends on their size, location, shape, degree of calcification and technical quality of the radiograph ${ }^{5)}$. However, normal anatomic structures, such as extra-pleural muscle and fat, may lead to false positive diagnosis in up to $20 \%$ of cases ${ }^{6}$.

On computed tomography (CT), plaques are readily recognized as circumscribed areas of pleural thickening with well-demarcated edges and are commonly located in the posterolateral and para-spinal regions of the tho- rax. Many studies showed that $\mathrm{CT}$ is more sensitive than CXR for the detection of pleural disease, especially for plaques located in the para-vertebral area $^{7,8)}$.

The aim of the present study is to improve the screening efficiency of CXR in identifying pleural plaques by proposing a plaque morphology-based classification.

\section{Subjects and Methods}

In this study, we hypothesized that the use of a morphology-based classification of pleural plaques, in addition to their location, width and extent with the other ILO criteria, may improve the detection rate of pleural plaque findings on CXR. The subjects of this study were 140 Japanese asbestos-exposed construction workers with probable and definite findings of pleural plaque on CXR. They were among current Japanese construction workers, and they underwent annual health check up during the period from 2005 to 2007 through the Mie Construction Workers' Health Insurance Society. The total number of the examined workers was 5,782, 5,771 and 5,346 in 2005, 2006 and 2007 respectively. One of the authors (N.H.) performed screening of their CXR regarding pleural plaque findings. A total of 311 workers with definite or probable findings of pleural plaque on CXR, were asked to perform chest $\mathrm{CT}$ scans. Among them, 179 workers agreed to continue in our research. Asbestos exposure was assessed using information obtained from the occupational histories of those workers. We excluded subjects with pulmonary pathological changes such as tuberculosis, diffuse pleural thickening and parenchymal fibrosis (irregular opacities corresponding to ILO category of $\geq 1 / 0$ ), which may interfere with pleural plaques detection on CXR and CT scans (Table 1). Moreover, cases with nonhomogenous shadows were also excluded in order to retain cases with probable or definite pleural plaques only (Fig. 1). Finally, the subject of this study was 140

Table 1. Diagnostic criteria of pleural plaque findings on CXR and CT scans

\begin{tabular}{|c|c|c|c|c|}
\hline & & \multicolumn{2}{|c|}{ CT Scans } & \multirow[b]{2}{*}{ Diagnosis } \\
\hline & & Lung tissue view & Mediastinal view & \\
\hline \multirow{7}{*}{ CXR } & \multirow{3}{*}{ In profile pleural plaques } & + & + & + \\
\hline & & + & - & \pm \\
\hline & & - & - & - \\
\hline & \multirow{2}{*}{ Face-on pleural plaques } & + & + & + \\
\hline & & - & - & - \\
\hline & \multirow{2}{*}{ Diaphragmatic pleural plaques } & + & + & + \\
\hline & & - & - & - \\
\hline
\end{tabular}

\pm No clear diagnosis of pleural plaques because only CT lung tissue views showed pleural plaque findings. 


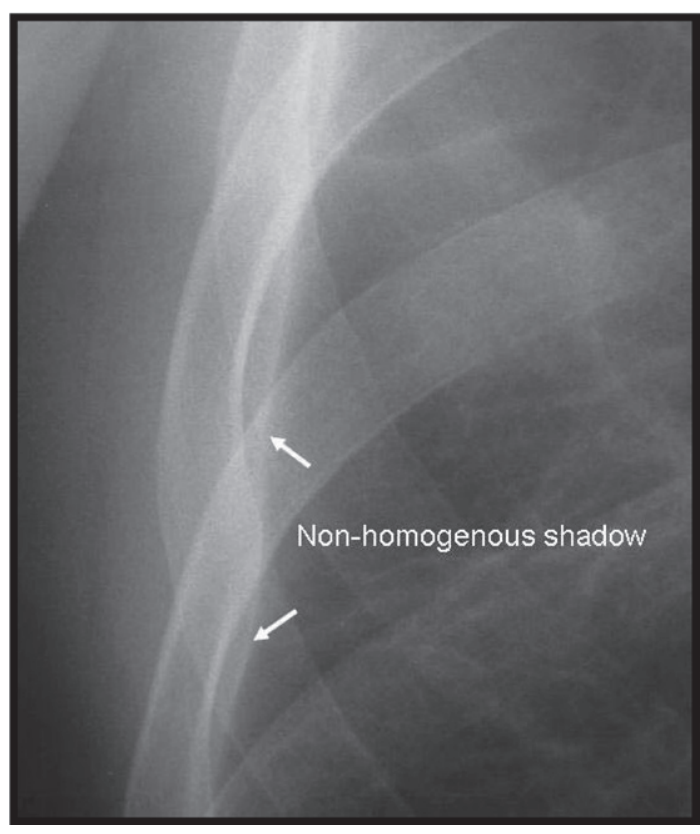

Fig. 1. Chest radiograph of an individual with nonhomogenous shadows as an example of the excluded cases.

workers. The site (chest wall and diaphragm), width, and extent of pleural plaque findings were recorded separately, according to the International Labour Office (ILO) classification of radiographs of pneumoconiosis 9). Moreover, all rib companion shadows were defined by shape for further classification.

Using CT scan as the gold standard for the diagnosis of pleural plaques, we evaluated the reading ability of CXR in detection of pleural plaques. The CXR and CT scans of each construction worker were coded and separated before being evaluated for pleural plaque shapes, sites, extent and width by three independent occupational physicians. The confirmed pleural plaques represented those that were agreed upon by consensus among the independent readings of them.

Throughout this research, we adhered to the Japanese ethical guidelines for epidemiologic studies; and the study protocol was approved by the Institutional Review Boards of Nagoya University Graduate School of Medicine.

\section{Results}

A total of 140 male construction workers were evaluated in this study; among them $65(46.4 \%)$ were carpenters, $15(10.7 \%)$ plasterers, $10(7.1 \%)$ steel frame workers, $9(6.4 \%)$ interior finishers, $7(5.0 \%)$ electricians, $6(4.3 \%)$ painters, $6(4.3 \%)$ plumbers, $6(4.3 \%)$ roofers, and $16(11.5 \%)$ had performed various other construction-related tasks. The mean age of the workers was $60.0 \pm 7.5 \mathrm{yr}$; and the majority (about $73.6 \%$ ) spent long periods (30-49 yr) in the construction-related jobs, during which they were exposed to asbestos (Table 2).

According to the ILO classification of radiographs of pneumoconiosis, among 92 subjects with chest wall pleural plaques (in-profile + face-on), 45 (49\%) showed plaques on both sides of the pleura, $16(17 \%)$ on the left side and $31(34 \%)$ on the right side. Among the 61 subjects with diaphragmatic pleural plaques, 18 (30\%) had plaques on both sides, $21(34 \%)$ on the left side and $22(36 \%)$ on the right side. Calcification was detected in 34 sites, 21 were located on the diaphragm (9 bilateral, 9 on the right and 3 on the left side), 9 were facing-on, 2 on the cardiac margins, and 2 on the chest wall (Table 3).

Examination of the $140 \mathrm{CT}$ scans showed 427 sites of pleural plaque; 203 in-profile chest wall, 43 faceon, 89 para-vertebral, 6 on the cardiac margins and 86 diaphragmatic pleural plaques. In 22 sites (9 in-profile, 4 face-on, 2 para-cardiac and 7 diaphragmatic sites), the CXR showed no lesion while chest CT scans demonstrated plaques (Table 4). Using CT as the gold standard, the frequency of CXR in-profile, face-on, paracardiac and diaphragmatic plaques confirmed on CT was 194 out of 203, 39 out of 43, 4 out of 6 and 79 out of 86 , respectively.

The percentage of calcification detected at the diaphragmatic, para-vertebral, facing-on and para-cardiac sites on CT was (30/86) 35\%, (15/89) 17\%, (23/43) $53 \%$ and (2/6) 33\%, respectively (Fig. 2).

The in-profile chest wall probable or definite pleural plaques were classified into nine types shown in Table 5. The following are the frequencies of in-profile pleural plaques on CT among pleural plaques on CXR according to their types: a straight medial border in 40 out of $43(93 \%)$ pleural plaque shadows, diamondshaped in 56/63 (89\%) pleural plaque shadows, doubleshape appearance in $7 / 8(88 \%)$ pleural plaque shadows,

Table 2. Frequency distribution of the workers according to the time spent working in the construction industry

\begin{tabular}{cc}
\hline $\begin{array}{l}\text { Time spent working in the } \\
\text { construction industry (years) }\end{array}$ & Number (\%) \\
\hline $\mathrm{n}$ & 140 \\
$<20$ & $2(1.4 \%)$ \\
$20-29$ & $13(9.3 \%)$ \\
$30-39$ & $56(40.0 \%)$ \\
$40-49$ & $47(33.6 \%)$ \\
$\geq 50$ & $19(13.6 \%)$ \\
Unknown & $3(2.1 \%)$ \\
Mean \pm SD & $39.6 \pm 9.0$ \\
\hline
\end{tabular}


Table 3. Pleural plaque findings according to ILO classification of pneumoconiosis, 2002

\begin{tabular}{|c|c|c|}
\hline Pleural plaques & $\mathrm{n}$ & $(\%)$ \\
\hline \multicolumn{3}{|l|}{ Site } \\
\hline \multicolumn{3}{|l|}{ In profile } \\
\hline Bilateral & 45 & 49 \\
\hline Right side & 16 & 17 \\
\hline Left side & 31 & 34 \\
\hline \multicolumn{3}{|l|}{ Face on } \\
\hline Bilateral & 7 & 44 \\
\hline Right side & 6 & 38 \\
\hline Left side & 3 & 19 \\
\hline \multicolumn{3}{|l|}{ Diaphragm } \\
\hline Bilateral & 18 & 30 \\
\hline Right side & 22 & 36 \\
\hline Left side & 21 & 34 \\
\hline \multicolumn{3}{|l|}{ Other site(s) } \\
\hline Left paracardiac & 4 & 100 \\
\hline \multicolumn{3}{|l|}{ Calcification } \\
\hline \multicolumn{3}{|l|}{ In profile } \\
\hline Bilateral & 0 & 0 \\
\hline Right side & 1 & 50 \\
\hline Left side & 1 & 50 \\
\hline \multicolumn{3}{|l|}{ Face on } \\
\hline Bilateral & 5 & 56 \\
\hline Right side & 3 & 33 \\
\hline Left side & 1 & 11 \\
\hline \multicolumn{3}{|l|}{ Diaphragm } \\
\hline Bilateral & 9 & 47 \\
\hline Right side & 9 & 42 \\
\hline Left side & 3 & 11 \\
\hline \multicolumn{3}{|l|}{ Other site(s) } \\
\hline Left paracardiac & 2 & 100 \\
\hline \multicolumn{3}{|l|}{ Extent } \\
\hline \multicolumn{3}{|l|}{ Right side } \\
\hline up to $1 / 4$ of the lateral chest wall & 35 & 57 \\
\hline $1 / 4$ to $1 / 2$ of the lateral chest wall & 21 & 34 \\
\hline$>1 / 2$ of the lateral chest wall & 5 & 8 \\
\hline \multicolumn{3}{|l|}{ Left side } \\
\hline up to $1 / 4$ of the lateral chest wall & 37 & 49 \\
\hline $1 / 4$ to $1 / 2$ of the lateral chest wall & 26 & 34 \\
\hline$>1 / 2$ of the lateral chest wall & 13 & 17 \\
\hline \multicolumn{3}{|l|}{ Width ( $3 \mathrm{~mm}$ minimum width) } \\
\hline \multicolumn{3}{|l|}{ Right side } \\
\hline 3 to $5 \mathrm{~mm}$ & 31 & 51 \\
\hline 5 to $10 \mathrm{~mm}$ & 25 & 41 \\
\hline$>10 \mathrm{~mm}$ & 5 & 8 \\
\hline \multicolumn{3}{|l|}{ Left side } \\
\hline 3 to $5 \mathrm{~mm}$ & 35 & 46 \\
\hline 5 to $10 \mathrm{~mm}$ & 34 & 45 \\
\hline$>10 \mathrm{~mm}$ & 7 & 9 \\
\hline
\end{tabular}

medially-tapered in 19/23 (83\%) pleural plaque shadows, parallel shaped (to the rib margin) in 20/25 (80\%) pleural plaque shadows, crescent-shaped in 23/33 (77\%) pleural plaque shadows, tenting appearance with base at the chest wall in 11/14 (79\%) pleural plaque shadows, tapered laterally (long type $>3 \mathrm{~cm}$ in length) in $18 / 25$ $(72 \%)$ pleural plaque shadows and tapered laterally (short type $\leq 3 \mathrm{~cm}$ in length) (Table 5). Pleural plaque shadows with straight medial border and diamond-shape border were the most common on the CXR, comprising 40 and 56 sites, respectively (Fig. 3).

We excluded data obtained from 33 in-profile sites since the diagnosis of pleural plaques was not clear. On the CXR of those 33 sites, we found pleural shadows, while there were no corresponding pleural plaque findings on CT mediastinal views; only CT lung tissue views showed pleural plaque changes. The percent of those excluded shadows that were confirmed only on CT lung tissue views among the whole diagnosed in-profile pleural plaques was $14 \%$ (33/240).

\section{Discussion}

In this study, we found that the use of a morphologybased classification of pleural plaques, in addition to their location, width and extent with other ILO criteria, will help pleural plaque identification and improve its reading on CXR. It seems that pleural plaque shadows with straight medial border and diamond-shape were the most common on the CXR and have the highest percentages of confirmed pleural plaques on $\mathrm{CT}$, while shadows which tapered laterally (short type) often appears unlikely in CT. Shadows that were ruled out to be pleural plaques on CT scans had the same CXR shape of the confirmed shadows but they were less in density and width. On the other hand, on CT scans, no corresponding signs could be found for 9 rib companion shadows that were detected on CXR with their thick parts directed medially (tapered laterally short type $\leq 3 \mathrm{~cm}$ in length). After applying our morphologybased classification of pleural plaques, CXR couldn't detect 9 sites of chest wall pleural plaques $(9 / 240=3.7 \%)$,

Table 4. Pleural plaque findings on CXR and CT scans

\begin{tabular}{lcclcrrr}
\hline & $\begin{array}{l}\text { Pleural } \\
\text { plaque find- } \\
\text { ings on CXR }\end{array}$ & $\begin{array}{l}\text { CT confirmed } \\
\text { pleural } \\
\text { plaques }\end{array}$ & $\begin{array}{l}\text { Falsely diag- } \\
\text { nosed pleural } \\
\text { plaques on CXR }\end{array}$ & $\begin{array}{l}\text { Falsely missed } \\
\text { pleural plaque } \\
\text { on CXR }\end{array}$ & $\begin{array}{l}\text { Total PP find- } \\
\text { ings on CT }\end{array}$ & $\begin{array}{l}* \text { SN } \\
(\%)\end{array}$ & $\begin{array}{c}* \text { PPV } \\
\text { In-profile-chest wall pleural plaques }\end{array}$ \\
Face-on pleural plaques & 240 & 194 & 46 & 9 & 4 & 203 & 96 \\
Para-cardiac pleural plaques & 44 & 39 & 5 & 2 & 83 & 91 & 89 \\
Diaphragmatic pleural plaques & 4 & 4 & - & 7 & 67 & 100 \\
\hline
\end{tabular}

*SN: Sensitivity; PPV: Positive Predictive value. 


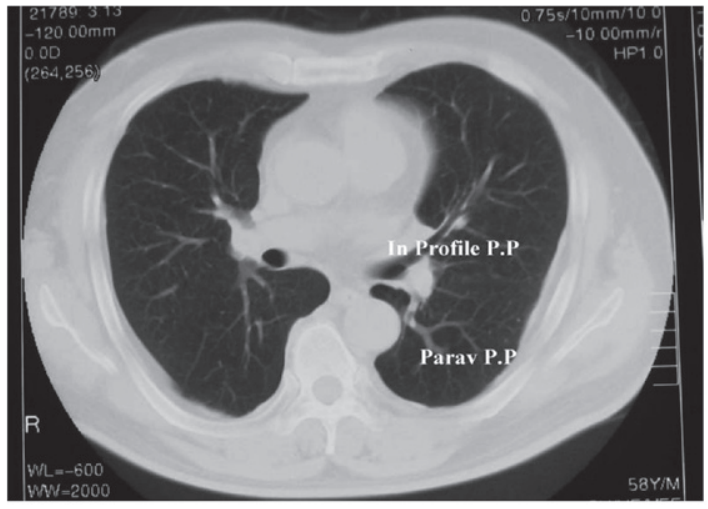

(a)

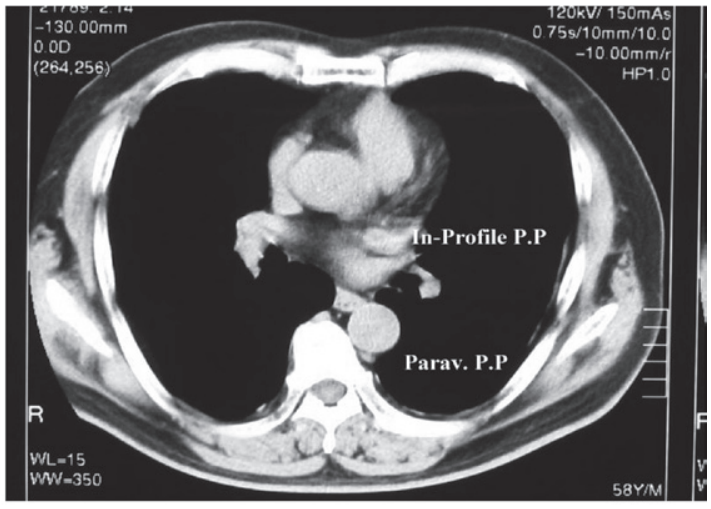

(b)

Fig. 2. Conventional CT images of the lung (a) and mediastinum (b) showing calcified in-profile and para-vertebral pleural plaques.

Table 5. Schematic representation and distribution of in-profile chest wall pleural plaque findings on CXR and their confirmed percentage on CT scans

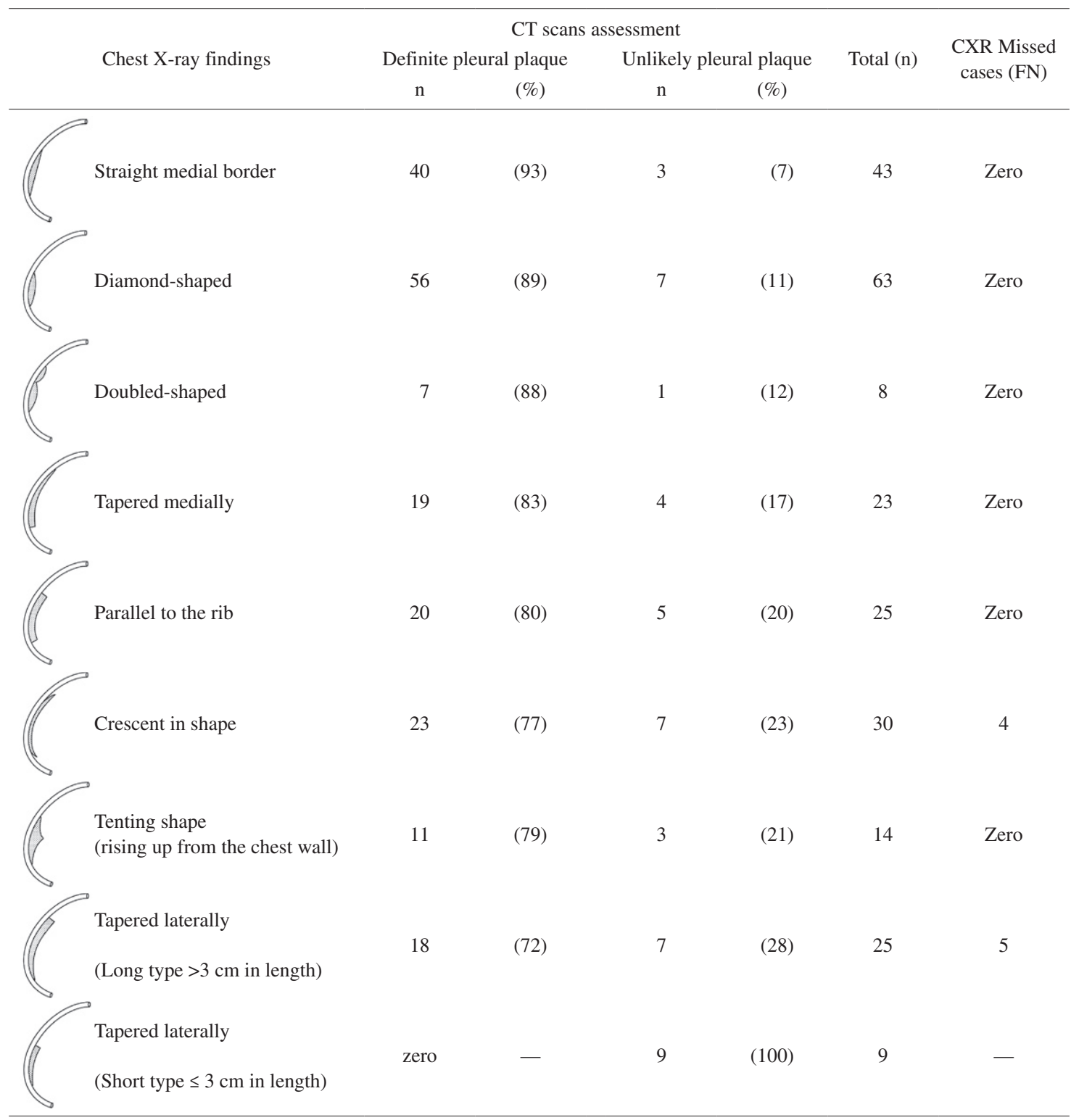



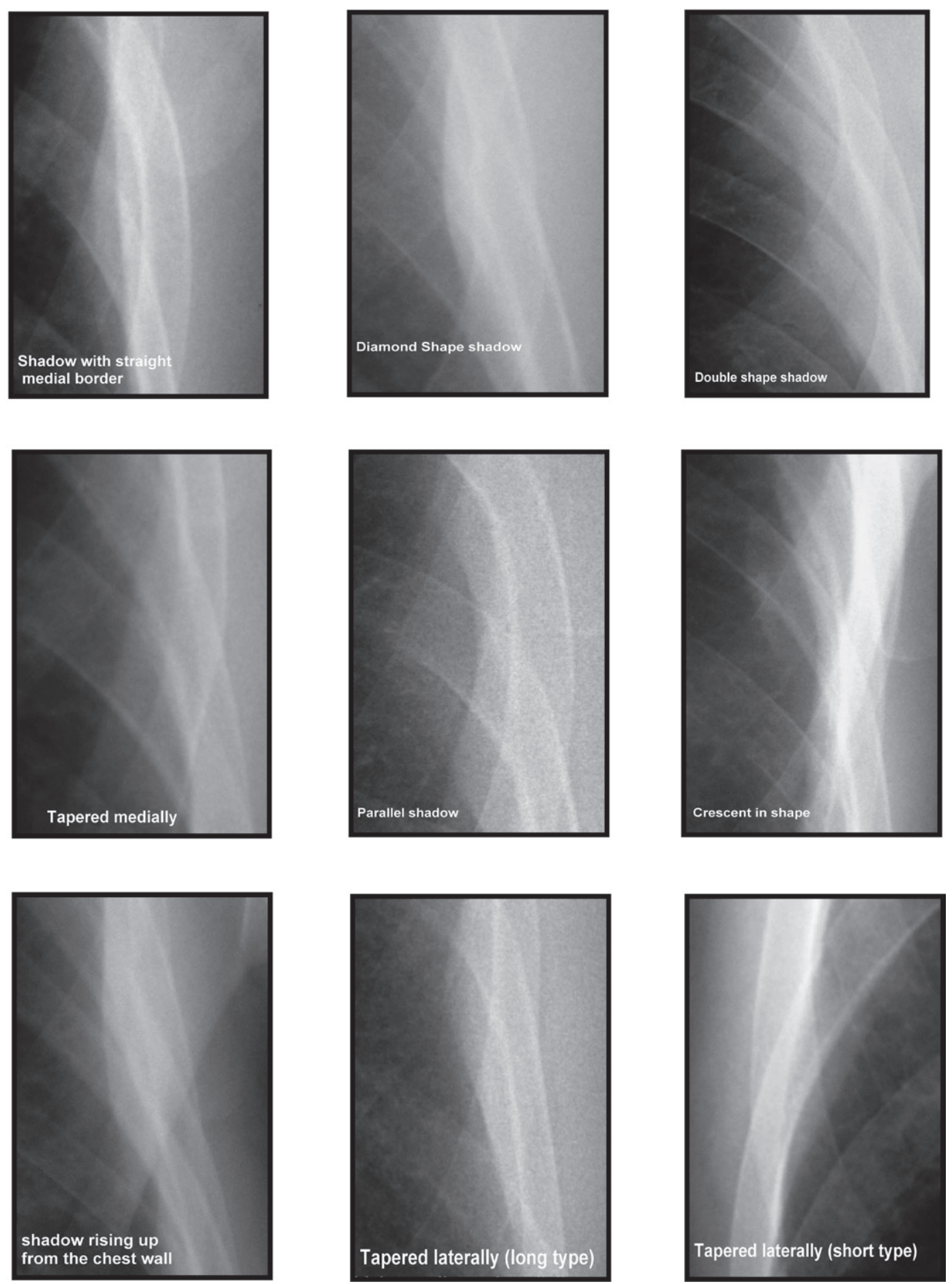

Fig. 3. Chest radiographs of construction workers exposed to asbestos showing different morphologies of the in-profile pleural plaques.

5 shadows have shadows tapered laterally (long type) and 4 shadows have crescent shape but their width is less than $2 \mathrm{~mm}$, taken together, these results suggest better sensitivity of CXR except the above mentioned two types of pleural plaque shadows and this could be a good way to decrease the false negative cases in CXR by exclusion of these shapes.

According to the ILO classification of pneumoconiosis radiographs, our results were consistent with the findings of Kikuchi and coworkers 9,10$)$ who reported that $68.6 \%$ out of 70 cases with chest wall pleural plaques (in-profile + face-on) were distributed on both 
sides of the chest, while $25.7 \%$ were on the left and $5.7 \%$ on the right. They also found that among 40 cases with diaphragmatic pleural plaques, $22.5 \%$ was bilaterally located, $60 \%$ on the left side and $17.5 \%$ on the right. In their 22 subjects with calcified plaques, calcification was found in 32 sites; including 26 on the diaphragm (13 on the left and 13 on the right side), 3 on cardiac margins and 3 on the chest wall.

Our results showed 22 false negative sites on the CXR (9 in-profile, 7 diaphragmatic, 4 face-on and 2 para-cardiac sites). The CXR showed no lesions, while the chest $\mathrm{CT}$ scans demonstrated hyaline plaques in all 22 sites. These results are similar to those reported by Kishimoto et al ${ }^{11}$.

In this research, data from 33 in-profile sites were excluded because no clear diagnosis of pleural plaques could be made. It is possible that these plaques were early-stage plaques and hence the difficulty in the diagnosis. Therefore, follow-up of those workers with CXR is recommended. Alternatively, the structures on the CXR could have been false positive findings related to normal anatomical structures (e.g., extra-pleural muscles and fat).

Comparisons of positive findings on both types of imaging (CXR and CT scan) showed that the reading of chest wall, diaphragmatic, face-on and para-cardiac plaques in CXR is quite similar to CT findings. In this regard Suganuma and coworkers ${ }^{13)}$ reported a sensitivity of 0.94 for the CXR and CT scan for pleural plaques detection. This could be a good reason to back up the screening of dust-exposed workers by radiographs, which can be done at a lower cost. Technological innovation has allowed accurate diagnosis of dust-related pulmonary diseases, but not all dust-exposed workers can benefit from these new diagnostic modalities. In that sense, simple and inexpensive diagnostic screening tests such as CXR should be further assessed to maximize their sensitivity.

We couldn't detect pleural plaques located in the paravetebral site on CXR, while CT examination revealed 89 para-vertebral pleural plaques, in this regards many studies have shown that $\mathrm{CT}$ is more sensitive for the detection of pleural disease especially for plaques located in the paravertebral area ${ }^{12,13)}$. Our results add further support to these early studies and showed that CT scan is better than CXR in detection of pleural plaques located in the para-vertebral areas. However, we should take into consideration that the additional information to be gained from $\mathrm{CT}$ evaluation must be balanced by the additional costs and time required especially in screening large numbers of dust-exposed individuals over the coming decades. Hence, the CXR will continue to be advantageous as the screening tool of choice for detec- tion of asbestos-related pleural plaques. In conclusion, while CT scan has its unique advantage in the detection of pleural plaques in the para-vertebral sites, the reading of pleural plaques in CXR can be improved by classifications that include morphology with anatomical location, width, extent as well as other ILO criteria. CXR will continue to be the primary survey imaging modality of choice because of its simplicity, wide availability, low cost and low radiation exposure.

\section{Acknowledgements}

We thank Mrs. Mizutani, from the Department of Occupational and Environmental Health, Nagoya University Graduate School of Medicine, for the generous help in drawing the schematic graphs of rib companion shadows.

\section{References}

1) Churg A (1982) Fiber counting and analysis in the diagnosis of asbestos-related disease. Hum Pathol 13, 381-92.

2) Hillerdal G, Lindgren A (1980) Pleural plaques: correlation of autopsy findings to radiographic findings and occupational history. Eur J Respir Dis 61, 315-9.

3) American Thoracic Society (1986) Medical Section of the American Lung Association: the diagnosis of nonmalignant diseases related to asbestos. Am Rev Respir Dis 134, 363-8.

4) Fletcher DE, Edge JR (1970) The early radiological changes in pulmonary and pleural asbestosis. Clin Radiol 21, 355-65.

5) Peacock C, Copley SJ, Hansell DM (2000) Asbestosrelated benign pleural disease. Clin Radiol 55, 422-32.

6) Gefter WB, Epstein DM, Miller WT (1984) Radiographic evaluation of asbestos-related chest disorders. Crit Rev Diagn Imaging 21, 133-81.

7) Friedman AC, Fiel SB, Fisher MS, Radecki PD, LevToaff AS, Caroline DF (1988) Asbestos-related pleural disease and asbestosis: a comparison of CT and chest radiography. AJR Am J Roentgenol 150, 269-75.

8) Lozewicz S, Reznek RH, Herdman M, Dacie JE, McLean A, Davies RJ (1989) Role of computed tomography in evaluating asbestos related lung disease. Br J Ind Med 46, 777-81.

9) International Labour Office. Guideline for the use of ILO international classification of radiographs of pneumoconiosis, revised edition. http://www.ilo.org/ public/libdoc/ilo/2002/102B09_423_engl.pdf. Accessed March 25, 2003.

10) Kikuchi K, Hiraga Y, Yamamoto A (1986) Pleural thickening caused by asbestos exposure-relation between computed tomography and plain film. Nihon Kyobu Shikkan Gakkai Zasshi 24, 101-8 (in Japanese with English abstract). 
11) Hosoda Y, Hiraga Y, Sasagawa S (2008) Railways and asbestos in Japan (1928-1987) —epidemiology of pleural plaques, malignancies and pneumoconioses. J Occup Health 50, 297-307.

12) Kishimoto T, Morinaga K, Kira S (2000) The prevalence of pleural plaques and/or pulmonary changes among construction workers in Okayama, Japan. Am J Ind Med 37, 291-5.

13) Suganuma N, Kusaka Y, Hiraga Y, Hosoda Y, Shida H, Morikubo H, Matsumoto T (2001) Asbestos-related pleural abnormalities detected by chest x-ray: fair agreement with detection by computed tomography. J Occup Health 43, 365-70.

14) Aberle DR, Gamsu G, Ray CS (1988) High-resolution CT of benign asbestos-related diseases: clinical and radiographic correlation. AJR Am J Roentgenol 151, 883-91.

15) Aberle DR, Gamsu G, Ray CS, Feuerstein IM (1988) Asbestos-related pleural and parenchymal fibrosis: detection with high-resolution CT. Radiology 166, 729-34. 